\title{
10
}

\section{An application of fuzzy logic to student modelling}

\author{
Panagiotou Mihalis \\ Grigoriadou Maria \\ University of Athens \\ Greece
}

\begin{abstract}
In this paper we discuss the principles of the design of a student model module within an educational teaching program in the domain of vertical projectile motion. Taking into account knowledge in the students as well as their individual learning skills the model allows steering of the educational process through the use of fuzzy logic and quantitative measurements. The teacher can still adapt the teaching strategy to specific needs of the student. The model has a decision component which chooses the teaching strategy on the basis of recorded history. A prototype program of the 'Fuzzy student model' is described.
\end{abstract}

Main conference themes: artificial intelligence, tutoring

Educational areas: secondary education

Study topics: science/engineering, mathematics

Secondary keywords: assessment/testing, expert systems, learning models, modelling, multimedia 


\section{INTRODUCTION}

One of the advantages of computer based approaches in teaching is the one-toone correspondence between students and machines facilitating individualization of learning. An Intelligent Computer Assisted Instruction system (ICAI-system) should continuously adapt its teaching style to the needs of the student in accordance with the knowledge domain [1].

Of central importance is the understanding of the problems with which the student currently is confronted. Offering appropriate information which is comprehensible to the student at the right time, generating suitable problems, providing useful feedback, maintaining motivation, etc., require a description of the current state of knowledge of the student. One of the original goals of Intelligent Tutoring Systems (ITSs) was to extend the power and accuracy of the available instruction by building student models permitting selection of an appropriate teaching action. As John Self (1988) pointed out "grand ambition to build high fidelity student models can easily obscure the fact that, in practical terms, student models by themselves achieve nothing. Student models are merely data for the tutoring component of ITSs" [2]. Instructional planning has as goal to configure the most efficient sequence and presentation of the subject material and relies on accurate modelling of student preferences and learning styles. Student modelling is a form of assessment which was developed to help computer based tutoring systems decide which exercise to give to a student, when to interrupt, what level of explanation to give and so on [3]. Basically the crucial task of an adaptive teaching system is to determine its next action according to the student's previous behaviour.

Most of the approaches of student modelling (overlay modelling, perturbation modelling, bounded user modelling, etc.) involve a rather simplistic model of the learning process (not far removed from route learning). This type of model takes no account of the rich range of learning styles and capabilities which according to psychological evidence exist. It also ignores the individual differences between the students. Moreover neither the teacher nor the student can interfere with the student model to adapt these to specific learning styles.

Students do not always solve problems, develop goals, plans and strategies as finite automata where the state determines what happens next. The student model needs quite specific information about a student's prior knowledge and, if possible, specific needs [2].

In this paper we present a framework for the development of a student model which we call 'fuzzy student model'. This model has a two levels structure. Firstly it is a fine grain model for deciding for example which exercise to pick next or what hint to give to the student during the interaction between the student and the program, on the basis of whether the student has 
mastered a particular rule, concept or subskill. Secondly it works as a large grain model to reflect whether the student has mastered a more complicate piece of knowledge, for example a whole area of the knowledge domain. This can be done by observing a student work over a long time and gaining an accurate model of the particular strengths and weaknesses of the student [3]. The model creates a history of a tutoring session and uses these histories for adapting the teaching strategy to the particular needs of the student. The model allows teacher or the student to interfere in it; the idea being that the ITS should be more collaborative than just a tutor [2].

A tutoring program with multiple presentations of vertical projectile motion using this fuzzy student model has been developed. In this program both the state of knowledge of the student and the student's individual learning skills are taken into account when steering the educational process, using fuzzy logic theory on quantitative measurements.

\section{GENERAL PRINCIPLES}

It is a well established fact that there are significant individual differences in school performance due to differences in prior knowledge and learning skills. Ideally these should be modelled so that an ITS may present the domain to be taught in a way appropriate to the individual's learning abilities and perhaps remedy weaknesses in those abilities [4]. In order to achieve this, the student model must record information with respect to [5]:

- knowledge status (knowledge state, misconceptions, ...);

- skill status (reading level, level of mathematics, ...);

- cognitive capabilities (memory limitations, perspicacity, learning speed, learning performance, attention, generalization abilities, ...);

- metacognitive skills (understanding, use of the feedback, understanding of functional relationships).

All this information can be extracted from a learner through interrogation. When learners work to master a subject, they acquire or improve knowledge and skills. These can be mapped into the student model. Questions can be classified as [6]:

Fact Oriented Questions: Questions to test the knowledge of elementary facts and topic aspects directly related to the content of the curricular lesson.

Higher Order Comprehension Questions: Questions in order to test the understanding of conceptual and semantic units of the lesson. 
Generalization Questions: These questions test comparison, differentiation, abstraction and generalization abilities.

Questions for Recognition of Functional Interrelations: This type of question tests the knowledge and understanding of functional relationship.

Each question is connected with one or several knowledge elements from the subject area being studied and has a certain weight representing the importance or the complexity of question [7]. The analysis of the answers is the input to the student modelling process.

\section{DIAGNOSIS AND STUDENT MODELLING}

In general each element in the model is an uncertainty variable which can be treated as a fuzzy variable (in the sense of fuzzy set theory) creating a 'Fuzzy student model'. First we must create fuzzy subsets $A_{i}$ of a Universe of Discourse $U$ which are characterized by a membership function $\mu: U \rightarrow[0,1]$. This function associates with each element $y$ of $U$ a number $\mu_{A(y)}$ in the interval $[0,1]$ which represents the grade of membership of $y$ in $A$. The basis of $A_{i}$ is the set of points in $U$ where $\mu_{\mathrm{A}(\mathrm{y})}$ is positive. When the fuzzy subset $A$ has a finite basis $\left\{\mathrm{y}_{1}, \mathrm{y}_{2}, \ldots, \mathrm{y}_{\mathrm{n}}\right\}$ it can be represented in the form:

$$
\mathrm{A}_{\mathrm{i}}=\mu_{1} / \mathrm{y}_{1}+\mu_{2} / \mathrm{y}_{2}+\ldots \ldots+\mu_{\mathrm{n}} / \mathrm{y}_{\mathrm{n}}
$$

or

$$
A_{j}=\sum_{j=1}^{n} \mu_{j} / y_{j}
$$

in which $\mu_{\mathrm{j}}, \mathrm{j}=1, \ldots, \mathrm{n}$.

It should be noted that the "+"-sign designates the union rather than the arithmetic sum. A finite Universe of Discourse $U=\left\{\mathrm{y}_{1}, \mathrm{y}_{2}, \ldots, \mathrm{y}_{\mathrm{n}}\right\}$ may be therefore be represented simply by [8]:

$$
\mathrm{U}=\left\{\mathrm{y}_{1}+\mathrm{y}_{2}+\ldots+\mathrm{y}_{\mathrm{n}}\right\}
$$

Let us consider the process of creating the fuzzy set of estimated learner's knowledge. The knowledge is associated with a number of questions $(\mathrm{Q})$ and a set of possible answers (A) for each question [7]. The knowledge level is unsatisfactory, satisfactory, good or excellently corresponding to the Universe of Discourse U (these are the elements y of $U$ ). 
Each answer is associated with a membership function $\mu: U \rightarrow[0,1]$. This function associates with each element $y$ of $U$ a number $\mu_{\mathrm{A}(\mathrm{y})}$ in the interval $[0,1]$ which represents the grade of membership of $\mathrm{y}$ in $\mathrm{A}$, the degree of knowledge of the element being tested. So each answer $A_{j}$ is associated with a fuzzy set:

$$
A_{j}=y_{1} / \text { unsatisfactory }+y_{2} / \text { satisfactory }+y_{3} / \text { good }+y_{4} / \text { excellent }
$$

in which $\mathrm{y}_{\mathrm{i}} \rightarrow[0,1]$

As mentioned before the questions have a certain weight representing 'importance' or complexity of question. So the fuzzy set associated with each question $Q_{i}$ will be weighted by a weight vector $w_{i}=\left\{w_{i j}\right\}$ where $w_{i j}$ is a weight of answer $A_{j}$ for question $Q_{i}$. For assigning different weights to these fuzzy subsets which express their relative importance, we use the method proposed by T. L. Saaty which is based on subjective pairwise comparison of the importance of the objects [9].

Furthermore we use compositional rules which combine the weighted fuzzy subsets $A_{j}$ to find the final fuzzy subset $A$ for the knowledge level. For this fuzzy subset $A$ an algorithm is needed to make the assessment for the final characterization. The most effective algorithm is the rule of the preponderant alternative which, simply stated, selects the alternative with the highest value. In the case where the values in the Fuzzy set are bimodal or cover a wider range, it is recommended to select a characterization midway between the two peaks or at the centre of the range (centre of area approach) if possible.

In this approach one does not know 'exactly' which state the student is in. Such 'exact' knowledge is in general not needed (certainly human teachers do not need it); it suffices to decide from the answers to the questions the 'knowledge zone' the student is in.

Estimation of the individual skills or weaknesses of student is done by the following method. These learning skills and weaknesses which our system tries to detect, are qualitatively described. The information which can be recorded directly through interrogation of the student, has a quantitative nature. Thus we have to find a way leading from quantitative measurements to qualitative results. In our view again fuzzy logic theory is appropriate for making the qualitative assessment of student skills.

In our model the quantitative measurements are: Time for answering, Total learning time needed, Number of attempts to find the correct answer, Kind of answer, Mistakes in using functions, Arithmetic mistakes, Number of repetitions (loops) of a topic until the student understands it, etc. [6]. All these are recorded during interrogation of the student through the different questions in the program and the values are used to assess the student's skills (learning 
speed, level of mathematics, attention, memory limitation, understanding of functional relationship abilities, etc.) [10].

The Universe of Discourse has as elements y values in the interval $[-3,3]$ where -3 corresponds to a lack of learning skill and 3 corresponds to presence of the skill; 0 reflects uncertainty about the skill, and all the other of values partial presence (positive) or partial lack (negative) in skill [11]. Each measurement $\mathrm{M}_{\mathrm{i}}$ is associated with a membership function $\mu: U \rightarrow[0,1]$. This function associates with each element $y$ of $U$ a number $\mu_{\mathrm{S}(\mathrm{y})}$ in the interval $[0$, 1] which represents the grade of membership of $y$ in $S_{i}$ where $S_{i}$ is the student's skill which the system can detect. For every measurement we have a fuzzy set $\mathrm{M}_{\mathrm{j}}$ corresponding to individual differences:

$$
\mathrm{M}_{\mathrm{j}}=\mu_{1} / \mathrm{y}_{1}+\mu_{2} / \mathrm{y}_{2}+\ldots \ldots+\mu_{\mathrm{k}-1} / \mathrm{y}_{\mathrm{k}-1}+\mu_{\mathrm{k}} / \mathrm{y}_{\mathrm{k}}
$$

in which $\mu_{1} \rightarrow[0,1]$ and $y_{i} \rightarrow[-3,3]$.

In order to find the final fuzzy set of individual differences and to then make the assessment of the characterization we use the same method (weights, etc.) as for defining the 'knowledge zone' of the student. This method is applied to every learning skill embedded in the system.

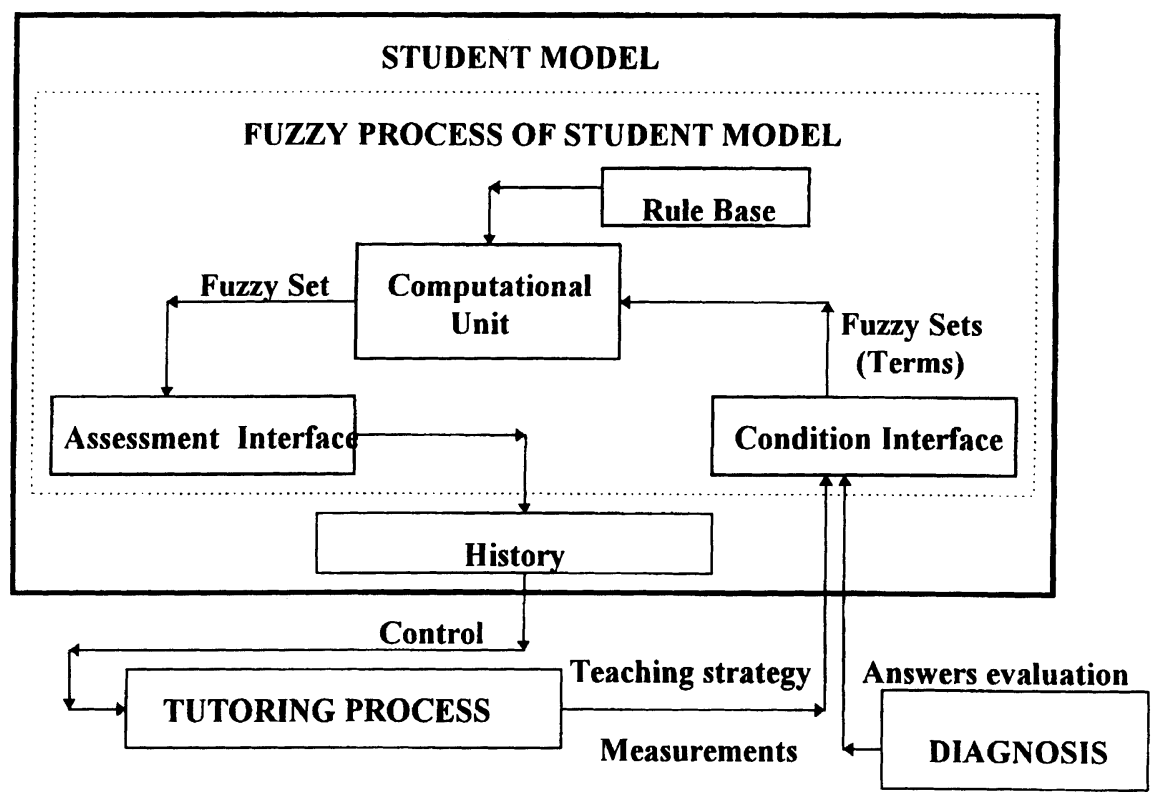

Fig. 1 Fuzzy student model 
Whenever a student finishes a subsession with a particular teaching strategy (presentation of content, number of questions, kind of questions, flow of the questions, etc.) the knowledge zone is characterized and the student's individual skills are assessed. The student may only go on with the tutoring session when characterization and assessment are satisfactory; in other cases the program will change its way of presentation of content, adding or removing theory and corresponding questions, changing the flow of the questions, etc. In our student model all these (teaching strategy, knowledge zone and the assessment of the student's individual skills) are recorded when the student finishes the subsession and are 'Events' of an 'Episode' of the 'History' of the student [12] (Fig. 1). All the episodes which take place during a tutoring session, create the 'session history' for the particular student and all session histories create the history of the student. The 'Episode' is more or less a short term memory because it is used only during the subsession and is only permanently recorded when the student has finished a satisfactory 'Episode' (fig. 2). The 'session history' works as a long term memory which is permanently recorded when the student finishes a tutoring session mastering a more complicate piece of knowledge, for example a whole area of the knowledge domain.

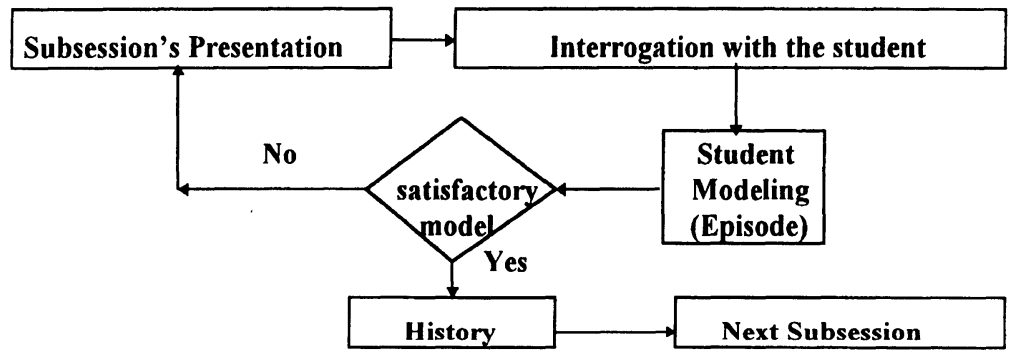

Fig. 2 Episode (short term memory)

On first contact a student is considered to be an average student by the system. But the teacher, or even the student after teacher permission, may change this. When the system is used by a student with a history the following operations may occur:

- Access of the history of the student (long term memory), decision of the most suitable teaching strategy and most appropriate hints to be given to the student.

- Comparison of the current episode (short term memory) with episodes of the already recorded history (long term memory) trying to identify similar episodes. In this case the system adopts the teaching strategy of the final 
session recorded in the history. This allows the system to arrive more quickly at the appropriate teaching strategy in less steps of adaptation.

In our system the characterizations, the fuzzy subsets which the system uses, and the weights represent the expertise of the teacher in defining the learning skills of the student. The events of an episode, the episodes of a session history and finally the whole history constitute the expertise of our system in defining the appropriate teaching strategy for the particular student.

Via a special interface the program is not only adaptable to the student's specific skills, but also to the teacher's personal views on the educational process. This makes the program a dynamic tool, not only for the teacher, but for the student as well (Fig. 3).

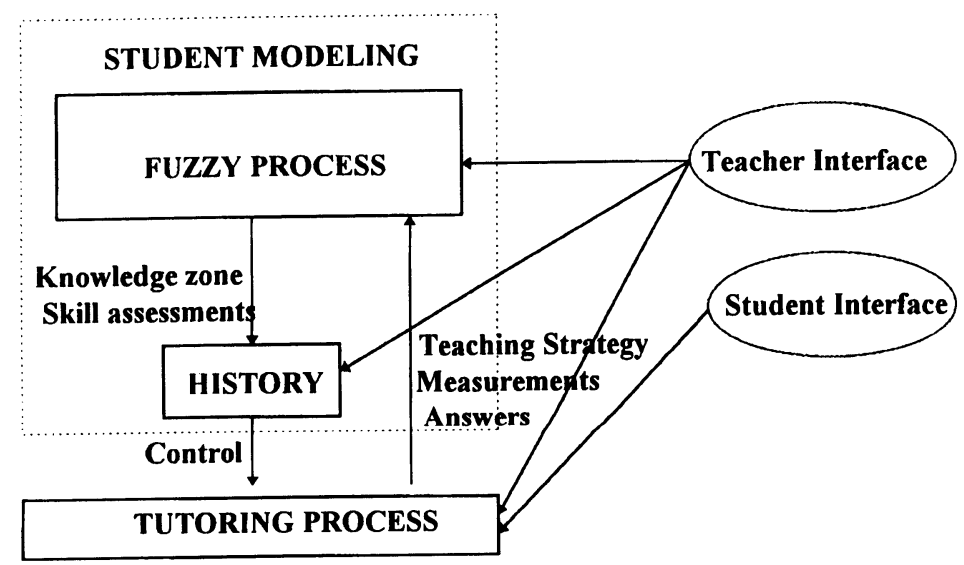

Fig. 3 Stages of teacher or student interference in our tutoring program

\section{PROTOTYPE IMPLEMENTATION}

The structure of the prototype program is based on information obtained from interviews with teachers and students, and on some results in research papers. The tutorial module consists of: the knowledge base of the domain to be taught, the diagnostic component which examines student answers, the student model (see above) and the decision component which decides on adaptation of the teaching strategy according to the student model.

The knowledge base of the domain can be presented in different ways (text, animation, video, sound-or combinations), with different amount of information (detailed - regular - brief), different sequences of animation and at 
the end different kinds of questions and exercises (Fact Oriented Questions, Higher Order Comprehension Questions, Generalisation Questions and Questions for Recognition of Functional Interrelations).

The diagnostic part decides on right or wrong answers on the basis of information drawn from the student and teacher interview protocols and tries to identify the kinds of mistakes (misconceptions, reading or writing mistakes, lack of skills in solving the motion's definitions etc.) for use in the student model.

The student model detects the knowledge zone of the student, the attention of the student, the learning speed, problems in calculations and the understanding of functional relationship abilities.

The decision component uses estimation rules to decide whether there is deviation from the optimum performance and the teaching strategy should be changed.

\section{CONCLUSIONS}

The program is under development, but after a first and narrow evaluation done by a small group (seven students and two teachers) we think that is offers an effective approach to adaptable intelligent tutoring programs on the basis of determining and using individual student differences, and adapting the teaching strategy and presentation of content to the individual needs of the students. Our prototype has been rather restricted. Further development should include more learning skills and teaching strategies to allow for better evaluation of our model.

\section{REFERENCES}

1. Dillenburg, P. (1988) A progmatic approach to student modelling: principles and architecture of PROTO-TEG. Intelligent Tutoring-System. Summer University Le Mans 88.

2. Self, J. (1988) Bypassing the intractable problem of student modelling. Intelligent Tutoring Systems. Montreal, Quebec, Canada: Université de Montreal.

3. Martin, J. and Vanlehn, K. (1993) OLAE: Progress toward a multiactivity, Bayesian student modeler. Proceedings of AI-ED 93. Edinburgh, Scotland. 
4. Panagiotou, M., Grigoriadou, M., Metaxaki, Chr. and Philokiprou, G. (1989) Modelling the student in a tutoring system. Proceedings of the 6th Int. Conf. on Techology and Education. Orlando. Vol. 2.

5. Nawrocki (1987) Maintenance in Training. Kearsley, G. (ed.) Artificial Intelligence and Instuction: Application and Methods. Addison-Wesley.

6. Mandl, H. and Lesgold, A. (1988) Learning Issues for Intelligent Tutoring Systems. New York Springer.

7. Bertels, K. (1994) A Dynamic View on Cognitive Student Modeling in Computer Programming. Journal of Artificial Intelligence in Education 5 (1).

8. Zadeh L. A. (1988) Fuzzy Logic. IEEE Computer, April 1988.

9. Novak, V. (1988) Fuzzy Sets and their Applications. Adam Higler.

10. Emberson, S. (1990) Diagnostic Testing by Measuring Learning Processes: Psychometric Considerations for Dynamic Testing. In Frederiksen, N. ,Glaser, R., Lesgold, A. and Saffo M. G. (eds.) Diagnostic monitoring of skill and knowledge acquisition. Hillsdale, NJ, Erlbaum.

11. Petrushin, V. and Sinitsa, K. (1993) Using Probabilistic Techniques for Learning Modelling. Proceedings of AI-ED 93. Edinburgh, Scotland.

12. Bobrow, D. G. (1984) Qualitative Reasoning about Physical Systems. North Holland. 